\title{
Transition between Mechanisms of Laser-Induced Field-Free Molecular Orientation
}

\author{
I. Znakovskaya, ${ }^{1}$ M. Spanner, ${ }^{2}$ S. De, ${ }^{3,4}$ H. Li, ${ }^{3}$ D. Ray, ${ }^{3}$ P. Corkum, ${ }^{2,5}$ I. V. Litvinyuk,${ }^{3,6}$ C. L. Cocke, ${ }^{3}$ and M. F. Kling ${ }^{1,3}$ \\ ${ }^{1}$ Max-Planck Institute of Quantum Optics, Hans-Kopfermann-Strasse 1, D-85748 Garching, Germany \\ ${ }^{2}$ Steacie Institute for Molecular Sciences, National Research Council of Canada, Ottawa, Ontario K1A OR6, Canada \\ ${ }^{3}$ J. R. Macdonald Laboratory, Physics Department, Kansas State University, 116 Cardwell Hall, Manhattan, Kansas 66506, USA \\ ${ }^{4}$ Saha Institute of Nuclear Physics, 1/AF Bidhannagar, Kolkata 700064, India \\ ${ }^{5}$ Joint Attosecond Science Laboratory, University of Ottawa and National Research Council of Canada, \\ 100 Sussex Drive, Ottawa, Ontario K1A 0R6, Canada \\ ${ }^{6}$ Centre for Quantum Dynamics and Australian Attosecond Science Facility, Griffith University, \\ Nathan, Queensland 4111, Australia
}

(Received 1 July 2013; published 18 March 2014)

\begin{abstract}
The transition between two distinct mechanisms for the laser-induced field-free orientation of $\mathrm{CO}$ molecules is observed via measurements of orientation revival times and subsequent comparison to theoretical calculations. In the first mechanism, which we find responsible for the orientation of $\mathrm{CO}$ up to peak intensities of $8 \times 10^{13} \mathrm{~W} / \mathrm{cm}^{2}$, the molecules are impulsively oriented through the hyperpolarizability interaction. At higher intensities, asymmetric depletion through orientation-selective ionization is the dominant orienting mechanism. In addition to the clear identification of the two regimes of orientation, we propose that careful measurements of the onset of the orientation depletion mechanism as a function of the laser intensity will provide a relatively simple route to calibrating absolute rates of nonperturbative strongfield molecular ionization.
\end{abstract}

DOI: 10.1103/PhysRevLett.112.113005

PACS numbers: $33.80 . \mathrm{Wz}, 32.80 . \mathrm{Rm}, 42.50 . \mathrm{Hz}$

Laser-induced field-free molecular alignment has become a routine tool in studies of ultrafast dynamics of small molecules [1], ranging from experiments on attosecond dynamics [2] and high-harmonic generation [3] to the investigations of coupled electronic-vibrational dynamics [4]. In this technique, a strong but nonionizing ultrafast laser field is used to give an impulsive torque to the molecules via the polarizability [1] and hyperpolarizability [5] interactions. After the pulse is over, the dominant axis of polarizability of molecules briefly aligns along the polarization direction of the laser as the molecules undergo quantum rotational revival dynamics, thereby effectively allowing experiments to be carried out in the molecular frame. However, for all its success, laser-induced alignment does not differentiate between the two different polarities of polar molecules. Thus, when applied to polar molecules, for example, $\mathrm{CO}$, the bond axis can be aligned in space but the direction in which the $\mathrm{C}$ or $\mathrm{O}$ ends point is not controlled. Achieving control over this latter property, a process called molecular orientation, has proved to be a most challenging experimental task.

Field-free orientation was initially generated using a combination of lasers and static electric fields [6-8]. It was only recently that an all-optical, and hence simpler, technique for laser-induced field-free molecular orientation had been demonstrated first in Refs. [9,10] followed by Ref. [11]. These all-optical studies introduced a controversy into the literature. Although the experiments all made use of a similar two-color pump pulse, comprised of the fundamental frequency $\omega$ and its second harmonic $2 \omega$, they invoked differing theoretical interpretations to explain the underlying mechanism leading to orientation. The first two studies $[9,10]$ attributed the orientation to the hyperpolarizability (HP) interaction [12], while the third [11] claimed that an ionization depletion (ID) mechanism [13] was active. The present work resolves this inconsistency by experimentally demonstrating that in fact either mechanism can be active, depending on the intensity of the pump pulse, and therefore gives a complete and unified description of two-color laser-induced orientation.

We report on the experimental observation of a transition between the two proposed mechanisms. We present intensitydependent measurements of the laser-induced orientation of $\mathrm{CO}$ and compare them to recent theoretical predictions by Spanner et al., considering both the HP and the ID mechanisms [13]. By comparing the measured and calculated temporal structures of the revivals of orientation, we unambiguously assign the regimes of the HP and ID mechanisms. This assignment, based on the temporal structure of the revivals, is further supported by the intensity dependence of the maximum orientation, which displays a sharp change in slope as the ID mechanism becomes active. For CO molecules, we find that at intensities below $8 \times 10^{13} \mathrm{~W} / \mathrm{cm}^{2}$, the HP mechanism is responsible for orientation, while the ID mechanism becomes dominant at higher intensities. In addition, we find that the ID mechanism is responsible for generating the highest degrees of orientation we observe.

The two-color method of laser-induced orientation uses a pump pulse created by combining a linearly polarized pulse at its fundamental $(\omega)$ and second harmonic frequencies 
$(2 \omega)$. The symmetry along the polarization axis of the resulting field $E(t)=E_{\omega}(t) \cos (\omega t)+E_{2 \omega}(t) \cos (2 \omega t+\varphi)$ depends on the phase delay $\varphi$ between the two frequency components. Breaking this symmetry is the key to the orientation of heteronuclear molecules. The experimental setup is detailed in Ref. [9]. Briefly, linearly polarized pulses with 45 fs duration at $800 \mathrm{~nm}$ are produced using a Ti:sapphire laser and are split into a pump and a probe arm of a Mach-Zehnder interferometer. In the pump arm, the second harmonic is created, temporally synchronized with the fundamental, and its polarization is made parallel to the polarization of the fundamental. A rotatable calcite plate serves to adjust the relative phase $\varphi$ between the two colors of the excitation field. The phase is calibrated by measurement of the phase-dependent ionization of CO [14]. Note that this method corrects an error of $\pi$ in the assignment of $\varphi$ reported by De et al., which was based on abovethreshold ionization [9]. The resulting field-asymmetric two-color pump pulses are focused onto a supersonic jet of $\mathrm{CO}$ molecules $\left(T_{\text {rot }} \approx 60 \mathrm{~K}\right)$ inside a velocity-map imaging spectrometer (VMIS) [15] by a spherical mirror $(f=75 \mathrm{~mm})$ placed at the rear side of the VMIS. The intensity of the two-color pump pulses was varied between $2.4 \times 10^{13}$ and $1.2 \times 10^{14} \mathrm{~W} / \mathrm{cm}^{2}$. Here, both colors had an equal intensity in the focus, which has been derived from first principles calculations and confirmed by cutoff measurements of electrons emitted from Xe for the individual color beams.

The degree of orientation and alignment induced by the pump pulse is typically characterized by the observables $\langle\cos \theta\rangle$ and $\left\langle\cos ^{2} \theta\right\rangle$, respectively, where $\theta$ is the angle between the molecular axis and the polarization direction of the laser. It is not trivial to directly measure the angle $\theta$. As in Ref. [9], we use a strong single-color $(800 \mathrm{~nm})$ probe pulse to multiply ionize and Coulomb explode the molecule. The angle $\theta$ was then approximated by the angle $\theta \approx \theta_{\exp }$ at which fragments arising from the Coulomb explosion are detected with the VMIS. The intensity of the probe pulse is $(2.6 \pm 0.6) \times 10^{14} \mathrm{~W} / \mathrm{cm}^{2}$. Similar to the studies reported in Ref. [9], we have chosen to analyze the angular emission of $\mathrm{C}^{2+}$ fragments at kinetic energies above $2.5 \mathrm{eV}$. The top row of Fig. 1 shows the experimental
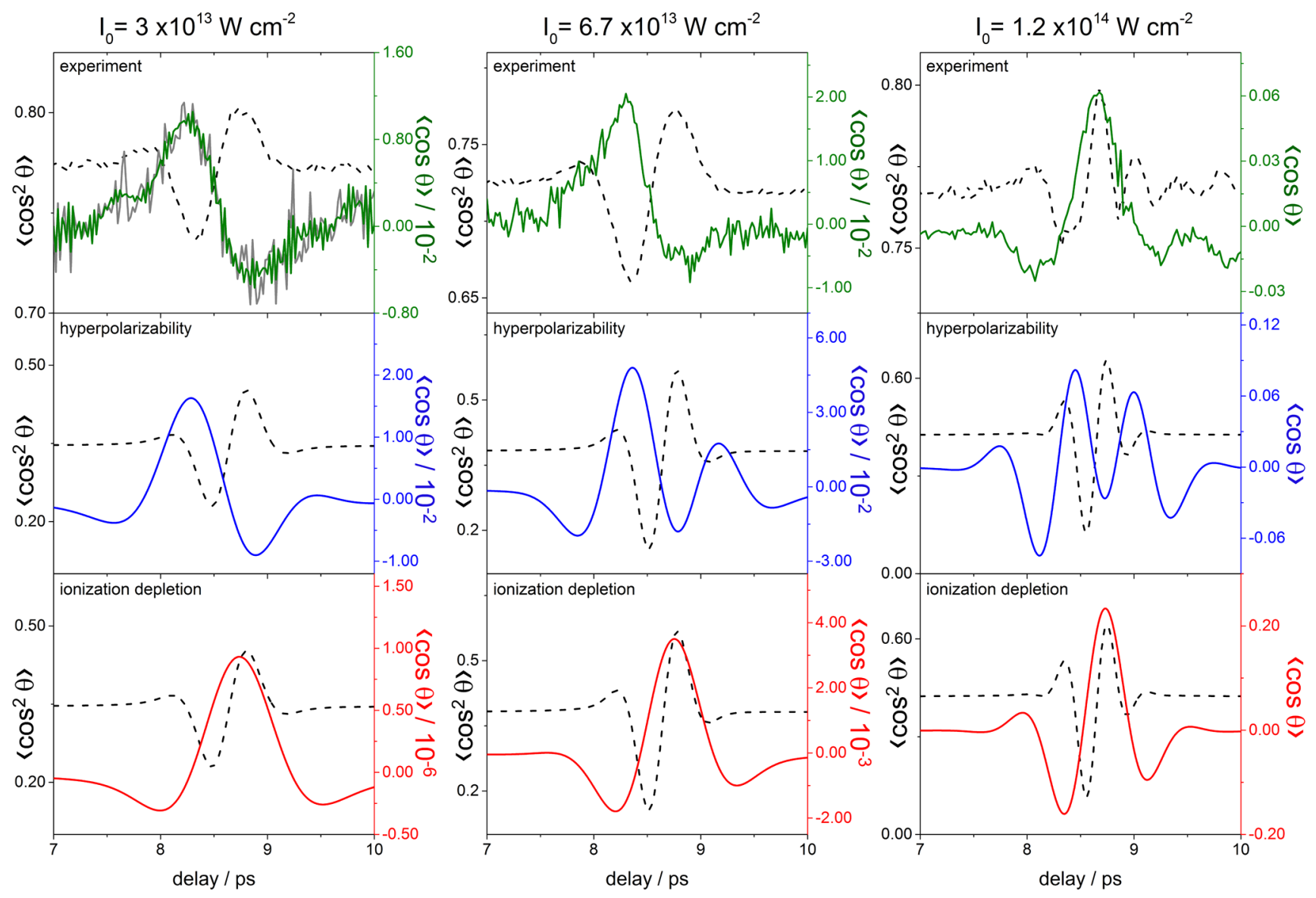

FIG. 1 (color online). Comparison of experimental dynamic alignment and orientation data for CO at three selected intensities of the two-color field (top row) with theoretical predictions from the hyperpolarizability (middle row) and ionization depletion mechanisms (bottom row). The alignment (dashed black lines in all rows) is represented by the parameter $\left\langle\cos ^{2} \theta\right\rangle$. The orientation is given by the parameter $\langle\cos \theta\rangle$. The experimental orientation data at the lowest intensity have been smoothed by a band-block Fourier filter $(2-19 \mathrm{THz})$. The original data are shown in grey. 
alignment traces (dashed black lines) around the first full revival of $\left\langle\cos ^{2} \theta\right\rangle$ near the molecules' full rotation time $1 /(2 B c)$ of 8.64 ps (with $B=1.93 \mathrm{~cm}^{-1}$ ) [16] at three selected two-color pump intensities. Also shown are the corresponding experimental orientation traces (green lines), which are reflected here by the parameter $\langle\cos \theta\rangle$. We note that the linearly polarized probe pulse increases the measured degree of alignment. Furthermore, since the probability of ionizing to the $\mathrm{C}^{2+}$ fragment as a function of the actual $\theta$ is not rigorously known, both the alignment and orientation values extracted from the Coulomb explosion will be proportional to, but not exact representations of, the $\langle\cos \theta\rangle$ and $\left\langle\cos ^{2} \theta\right\rangle$ observables.

We now turn to the theoretical description of the orientation mechanisms. For the computations, the twocolor laser pulse is written as

$$
E(t)=E_{0} f(t)[\cos (\omega t)+\cos (2 \omega t)],
$$

where $f(t)$ is the pulse envelope and $E_{0}$ is the peak electric field strength. In this definition of the $E(t)$, we have set the relative phase between the colors to zero: $\varphi=0$, which assumes the maximum field asymmetry. We choose the envelope function $f(t)$ to be

$$
f(t)= \begin{cases}0 & t<0 \\ \sin \left(\pi t / 2 \tau_{\text {on }}\right) & 0<t<2 \tau_{\text {on }} \\ 0 & t>2 \tau_{\text {on }},\end{cases}
$$

corresponding to a $\sin ^{2}$ pulse for the intensity $I=E^{2}$. The parameter $\tau_{\text {on }}$ is the full width at half-intensity, which we set to $\tau_{\text {on }}=45$ fs.

The rotational motion of the molecules does not follow the carrier oscillations of the laser, and hence it is appropriate to use the cycle-averaged Hamiltonian of the system to compute the rotational dynamics

$$
H(\theta, t)=B J(J+1)+V_{P}(\theta, t)+V_{H}(\theta, t)+V_{I}(\theta, t),
$$

where $B$ is the rotational constant, $V_{P}(\theta, t)$ is the polarizability term that generates molecular alignment [17], $V_{H}(\theta, t)$ is the hyperpolarizability term, and $V_{I}(\theta, t)$ accounts for ionization [13]. All equations use Hartree atomic units $\left(m_{e}=e=\hbar=1\right)$. For the particular $E(t)$ chosen in Eq. (1), the first two potential terms in Eq. (3) are given by [12]

$$
\begin{gathered}
V_{P}(\theta, t)=-\frac{1}{2} \Delta \alpha E_{0}^{2}|f(t)|^{2} \cos ^{2} \theta, \\
V_{H}(\theta, t)=-\frac{3}{8} \beta_{x x z} E_{0}^{3}|f(t)|^{3} \cos \theta \\
-\frac{1}{8}\left(\beta_{z z z}-3 \beta_{x x z}\right) E_{0}^{3}|f(t)|^{3} \cos ^{3} \theta,
\end{gathered}
$$

where $\Delta \alpha=\alpha_{\|}-\alpha_{\perp}$ is the polarizability anisotropy and the $\beta_{i j k}$ are elements of the hyperpolarizability tensor. The ionization depletion term is given by

$$
V_{I}(\theta, t)=-(i / 2) K(t) \Gamma_{\text {ref }}(\theta),
$$

where

$$
K(t)=\exp \left\{-\frac{2}{3}\left(2 I_{p}\right)^{3 / 2}\left[\left|E_{0} f(t)\right|^{-1}-\left|E_{\mathrm{ref}}\right|^{-1}\right]\right\}
$$

accounts for the tunneling exponent [18] that provides the dominant scaling of strong-field ionization, and

$$
\Gamma_{\text {ref }}(\theta)=c_{0}+c_{1} \cos \theta+c_{2} \cos 2 \theta
$$

accounts for the angle dependence of the ionization rate. As outlined in Ref. [13], this analytical form of $V_{I}(\theta, t)$ is specific to $\mathrm{CO}$ and was constructed as a fit to purely numerical computations that used the method of Ref. [19]. It is a complex potential causing nonunitary quantum evolution that removes amplitude as a function of angle, which captures the effects of population loss due to ionization. All the molecular constants that appear in the potentials are collected in Table I. Although the Hamiltonian [Eq. (3)] includes both the hyperpolarizability and ionization terms, we present results for each mechanism separately in order to clearly elucidate the characteristic features of the two mechanisms.

The time-dependent rotational Schrödinger equation for the Hamiltonian in Eq. (3) is solved in a spherical harmonics basis using the Crank-Nicholson method [21]. We account for the thermal distribution by propagating each initial rotational state $|J, M\rangle$ independently and then incoherently averaging the $\left\langle\cos ^{2} \theta\right\rangle$ and $\langle\cos \theta\rangle$ observables weighted by the Boltzmann distribution at temperature $T=60 \mathrm{~K}$. For the ionization depletion mechanism, the observables are further normalized to the remaining neutral population.

The results of our calculations for the HP (ID) mechanism are shown in the middle (bottom) row of Fig. 1, where alignment is displayed as dashed black lines and orientation as blue (red) lines. It can be seen that the temporal structure of the measured orientation revival is reflected well by the HP mechanism for the two lower intensities of $3 \times 10^{13}$ and $6.7 \times 10^{13} \mathrm{~W} / \mathrm{cm}^{2}$. At these two intensities, the computed fraction of ionized molecules is very low, $1.6 \times 10^{-4} \%$ for $3 \times 10^{13} \mathrm{~W} / \mathrm{cm}^{2}$ and $0.79 \%$ for $6.7 \times 10^{13} \mathrm{~W} / \mathrm{cm}^{2}$. Consequently, the ID mechanism does not yet yield a significant orientation signal compared to the signal from

TABLE I. Molecular constants (a.u.) used to model CO.

\begin{tabular}{lccc}
\hline \hline Parameter & Value [Reference] & Parameter & Value [Reference] \\
\hline$B$ & $8.7997 \times 10^{-6}[16]$ & $\Delta \alpha$ & $3.6[20]$ \\
$\beta_{z z z}$ & $28.91[20]$ & $\beta_{x x z}$ & $7.69[20]$ \\
$I_{p}$ & $0.516[16]$ & $c_{0}$ & $0.2214 \times 10^{-3}$ \\
$E_{\text {ref }}$ & 0.0535 & $c_{1}$ & $-0.2141 \times 10^{-3}$ \\
& & $c_{2}$ & $0.0822 \times 10^{-3}$ \\
\hline \hline
\end{tabular}


the HP mechanism. The situation changes at the highest intensity investigated of $1.2 \times 10^{14} \mathrm{~W} / \mathrm{cm}^{2}$. Here, the model predicts significant ionization $(36 \%)$ and the ID mechanism creates an orientation signal that is stronger than the one generated from the HP mechanism. In good agreement with the theoretical prediction, the experimental orientation trace resembles the orientation revival predicted by the ID mechanism. Remarkably, the transition between the mechanisms is well reflected in a temporal shift of the dominant orientation peak from around 8.25 and $8.3 \mathrm{ps}$ at $3 \times 10^{13}$ and $6.7 \times 10^{13} \mathrm{~W} / \mathrm{cm}^{2}$, respectively, to $8.7 \mathrm{ps}$ at $1.2 \times 10^{14} \mathrm{~W} / \mathrm{cm}^{2}$. Close inspection of the timing of the maximum of the orientation revival is thus a good indicator of the active orientation mechanism.

As further evidence for the transition between the mechanisms, Fig. 2 shows the measured maximum orientation values $|\langle\cos \theta\rangle|_{\max }$ (green dots) as a function of the two-color pump intensity. The horizontal error bars reflect the uncertainty in the determination of the pump intensity and the vertical error bars the errors in the measured orientation values. The experimental data are compared to the predictions from the HP (blue triangles) and ID (red squares) mechanisms. The theoretical $|\langle\cos \theta\rangle|_{\max }$ values are scaled by a constant, intensity-independent factor to give best quantitative agreement with the experimental data. Since the analysis of the data in Fig. 1 suggests that the HP mechanism is dominant at low intensities, the theoretical data for the HP mechanism were scaled to fit best to the data below $8 \times 10^{13} \mathrm{~W} / \mathrm{cm}^{2}$. It is remarkable that the measured gradient of $|\langle\cos \theta\rangle|_{\max }$ with intensity is so well predicted by the HP mechanism in this regime, lending further support for this assignment to be correct. At the intensities above $8 \times 10^{13} \mathrm{~W} / \mathrm{cm}^{2}$, the experimental

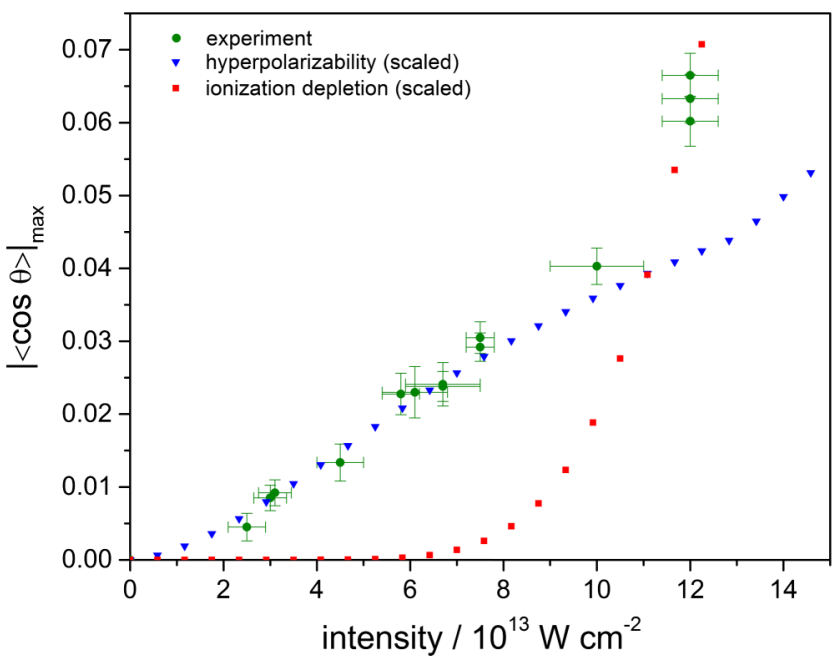

FIG. 2 (color online). Comparison of the measured intensity dependence of the maximum field-free orientation $|\langle\cos \theta\rangle|_{\max }$ to predictions for the hyperpolarizability and ionization depletion mechanisms. The theoretical data for the HP and ID mechanisms have been scaled by 0.51 and 0.27 , respectively. points diverge sharply from the HP curve and display a rapid increase. This rapid increase in the experimental maximum orientation matches qualitatively the exponential increase we would expect from the theoretical predictions of the ID mechanism.

The transition between the HP and ID mechanisms offers a unique opportunity for the calibration of strong-field ionization (SFI) rates. At present, there is no reliable method of computing absolute SFI rates for molecules. Many approaches exist to calculate the molecular SFI, from tunneling and semiclassical models like MO-SFA [22] and MO-ADK [23] to more numerically intensive methods like TD-DFT [24], TD-CIS [25], MCTDHF [26], and the mixed orbital-grid method of Ref. [19]. These methods have certainly provided much insight into molecular SFI and can explain reasonably well the general intensity scaling and angular dependence of molecular SFI. However, the ultimate reliability of these theories with respect to absolute ionization rates has not been tested. Although attempts to measure the absolute SFI rates of molecules can be found $[27,28]$, they are by no means routine. Detailed and well calibrated measurements of the transition between the HP and ID mechanisms provide a means to calibrate the strongly nonperturbative SFI response of polar molecules against the perturbative (and hence much better understood) hyperpolarizability response in one clean experiment. One can first ensure the modeling reproduces the orientation in the HP regime to yield a properly calibrated measurement, then extract the absolute SFI rate fitting the orientation in the ID regime. Since the uncharacterized angular probability of Coulomb exploding to the $\mathrm{C}^{2+}$ charge state presently prevents a quantitative extraction of the SFI rate, future work would need to focus on finding a more reliable experimental measure of $|\langle\cos \theta\rangle|_{\max }$.

In conclusion, we have studied the intensity-dependent transition between two mechanisms for the orientation of $\mathrm{CO}$ molecules in two-color laser fields. At low intensities, the HP mechanism is active, while at higher intensities, the ID mechanism dominates. We expect similar behavior for other polar molecules. Apart from reconciling the two differing mechanisms proposed in the literature, this assignment is important for the future applicability of the twocolor orientation technique. Since the highest degrees of orientation are reached via the ID mechanism, achieving large orientation will always entail the generation of large numbers of cations and free electrons in the sample. This necessary generation of cations and electrons that comes with large orientation could affect experiments using the two-color orientation technique, and it may be important to take them into account when using the technique as a tool in subsequent experiments. In addition to resolving the active mechanism in laser-induced molecular orientation, the observation of the transition between the two mechanisms offers a potential experimental observable that can be used to calibrate or extract the absolute strong-field 
ionization rates of molecules, which is presently a remaining computational challenge for all existing theories of strong-field ionization.

We are grateful for fruitful discussions with E. Frumker. We acknowledge support by the U.S. Department of Energy under DE-FG02-86ER13491, the DFG via $\mathrm{Kl}-1439 / 3$ and $\mathrm{Kl}-1439 / 5$, and the Cluster of Excellence: Munich Center for Advanced Photonics (MAP).

[1] H. Stapelfeldt and T. Seideman, Rev. Mod. Phys. 75, 543 (2003).

[2] F. Krausz and M. Ivanov, Rev. Mod. Phys. 81, 163 (2009).

[3] T. Kanai, S. Minemoto, and H. Sakai, Nature (London) 435 , 470 (2005).

[4] C. Z. Bisgaard, O. J. Clarkin, G. Wu, A. M. D. Lee, O. Geßner, C. C. Hayden, and A. Stolow, Science 323, 1464 (2009).

[5] S. Banerjee, D. Mathur, and G. R. Kumar, Phys. Rev. A 63, 045401 (2001).

[6] A. Goban, S. Minemoto, and H. Sakai, Phys. Rev. Lett. 101, 013001 (2008).

[7] O. Ghafur, A. Rouzee, A. Gijsbertsen, W. K. Siu, S. Stolte, and M. J. J. Vrakking, Nat. Phys. 5, 289 (2009).

[8] L. Holmegaard, J. L. Hansen, L. Kalhøj, S. L. Kragh, H. Stapelfeldt, F. Filsinger, J. Küpper, G. Meijer, D. Dimitrovski, M. Abu-samha et al., Nat. Phys. 6, 428 (2010).

[9] S. De, I. Znakovskaya, D. Ray, F. Anis, N. G. Johnson, I. A. Bocharova, M. Magrakvelidze, B. D. Esry, C. L. Cocke, I. V. Litvinyuk et al., Phys. Rev. Lett. 103, 153002 (2009).

[10] K. Oda, M. Hita, S. Minemoto, and H. Sakai, Phys. Rev. Lett. 104, 213901 (2010).

[11] E. Frumker, C. T. Hebeisen, N. Kajumba, J. B. Bertrand, H. J. Wörner, M. Spanner, D. M. Villeneuve, A. Naumov, and P. B. Corkum, Phys. Rev. Lett. 109, 113901 (2012).
[12] T. Kanai and H. Sakai, J. Chem. Phys. 115, 5492 (2001).

[13] M. Spanner, S. Patchkovskii, E. Frumker, and P. Corkum, Phys. Rev. Lett. 109, 113001 (2012).

[14] H. Li, D. Ray, S. De, I. Znakovskaya, W. Cao, G. Laurent, Z. Wang, M. F. Kling, A. T. Le, and C. L. Cocke, Phys. Rev. A 84, 043429 (2011).

[15] A. T. J. B. Eppink and D. H. Parker, Rev. Sci. Instrum. 68, 3477 (1997).

[16] NIST Chemistry Webbook, NIST Standard Reference Database, edited by P. J. Linstrom and E. W. G. Mallard (NIST, Gaithersburg, MD), Vol. 69, http://webbook.nist.gov (retrieved September 2011).

[17] B. Friedrich and D. Herschbach, Phys. Rev. Lett. 74, 4623 (1995).

[18] L. V. Keldysh, Sov. Phys. JETP 20, 1307 (1965).

[19] M. Spanner and S. Patchkovskii, Phys. Rev. A 80, 063411 (2009).

[20] K.A. Peterson and T.H. Dunning Jr., J. Mol. Struct. (Theochem.) 400, 93 (1997).

[21] W. H. Press et al., Numerical Recipes (Cambridge University Press, Cambridge, England, 1992), 2nd ed.

[22] J. Muth-Böhm, A. Becker, and F. H. M. Faisal, Phys. Rev. Lett. 85, 2280 (2000).

[23] X. M. Tong, Z. X. Zhao, and C. D. Lin, Phys. Rev. A 66, 033402 (2002).

[24] D. A. Telnov and S.-I Chu, Phys. Rev. A 79, 041401(R) (2009).

[25] N. Rohringer, A. Gordon, and R. Santra, Phys. Rev. A 74, 043420 (2006).

[26] M. Kitzler, J. Zanghellini, C. Jungreuthmayer, M. Smits, A. Scrinzi, and T. Brabec, Phys. Rev. A 70, 041401(R) (2004).

[27] A. S. Alnaser, X. M. Tong, T. Osipov, S. Voss, C. M. Maharjan, B. Shan, Z. Chang, and C. L. Cocke, Phys. Rev. A 70, 023413 (2004).

[28] C. Smeenk, J. Z. Salvail, L. Arissian, P. B. Corkum, C. T. Hebeisen, and A. Staudte, Opt. Express 19, 9336 (2011). 